CONFERENCE SUMMARY 


\title{
KEELE CATACLYSMIC COMMENTARY
}

\author{
BRIAN WARNER \\ Department of Astronomy, University of Cape Town, \\ 7700 Rondebosch, South Africa
}

What kind of person is invited to make Conference summaries? For conference introductions it's easy - you just invite some past, long-retired Directorsaurus Rex to make a few deathbed corrections to his memories of how he founded the field and anticipated all future developments. For conference after-dinner speeches it's easy to find a sharp-elbowed antagonist for whom a few more unexpected remarks and double entendres can't hurt an already notorious reputation. But in a conference summariser one is looking for even-handedness, wisdom, unsurpassed knowledge, ability to pick winners and, above all, brevity.

This is why conference summaries are an impossible dream; and why conference summarizers often resort to the antics of introducers or afterdinner speakers.

What I am about to say is more of a coda than summary. In a conference like the one we have experienced this week a summary could not do justice. The fact is, the field of CVs has become so large that much of what we have heard have been summaries of specialised areas, with glimpses of work in progress. Many fields have not been touched on at all. The posters fill in some but not all of the gaps. I was tempted to illustrate the point by providing a summary of everything not said - but realised that I was hardly qualified to speak for the silent majority.

What has become quite evident is that most progress continues to be the result of improvements in equipment power. Historians of science analyse scientists of the past centuries into 'instrumentalists' and others. Hipparchus and Galileo are examples of the former, Copernicus and Newton are examples of the latter.

Looking at the history of $\mathrm{CVs}$, observational techniques changed from visual and photographic provision of long-term light curves, through the spectroscopy and photoelectric photometry of the early 1960s which established that CVs were close binaries. Progress then came in a series of jumps, with the introduction of high time resolution photometry and spectroscopy in the late 1960 s, the impact of satellite UV and X-ray observations in the 
$1970 \mathrm{~s}$, the increased sensitivity of infrared detectors and the detection of CVs at radio wavelengths and eventually even at $\gamma$-ray energies.

The most important results at this conference have conspicuously come from the latest instrumental developments - from ROSAT, EUVE, ASCA and HST; from Merlin and VLA; and the promise of new types of behaviour to be found by ALEXIS. Equally important as the new hardware are the analysis techniques:- again some of the finest new results are from applications of eclipse deconvolution ${ }^{1}$ and Doppler tomogram methods to observational material of high signal-to-noise ratio.

It is now possible to image directly or indirectly the early stages of eruptions of novae, the distribution of emission measure in non-magnetic and magnetic CVs, the asymmetric distribution of absorption or emission line strengths over the surfaces of $\mathrm{CV}$ secondaries, the stream of gas propellered out of AE Aqr, the relativistic ejection of collimated blobs of gas from low mass X-ray binaries such as GRS $1915+105$, etc. Details are being learnt of the heating and subsequent cooling of the white dwarf primaries of dwarf novae in outburst; of the structure of CV winds; of the inhomogeneities of nova ejecta; of the field geometry and positions of accretion regions in polars. Possibilities have been raised of rapidly learning more about the X-ray emitting regions in polars and the boundary layers in non-magnetic CVs by examining the ratios of reflected to direct hard X-ray spectra from white dwarf and accretion disc surfaces. This may help to clear the confusion still existing in boundary layer structures - from most observational evidence, boundary layers are much cooler $\left(\sim 10^{5} \mathrm{~K}\right)$ and of larger extent than the classical predictions.

The last CV conference, in Cape Town, was very specialised. This one has allowed discussions in all fields, including related stars. I think that it is clear that both kinds are necessary in order to avoid the danger of losing the broad picture. CV observers will be envious of the richness and understanding of QPOs in low-mass X-ray binaries; LMXB observers must envy the ease with which the primary and central regions of the disc are observed in some CVs. Both are concerned about disc rim structures and the existence of emission in unexpected regions of discs.

The number of astronomers and the number of publications has been growing exponentially for some decades. Fortunately the increase in advanced instrumentation has largely kept pace. And there is more already on the horizon: the impact of 8-metre class telescopes and of AXAF and other satellites on the field of CV studies is going to be tremendous. The success of the High Speed Photometer on HST was such that we should agitate for

\footnotetext{
${ }^{1}$ If this technique has allowed some astronomers to see further into CVs it is because they have looked over the shoulders of dwarfs.
} 
its reinstatement. VLBI from space can hope to image the structures of the nearest CVs.

Let me now touch on a few subjects where I think I may make useful comments or ask helpful questions. These serve to establish that I was conscious for many of the sessions.

1. It seems to me clear that there are two sources of flickering in nonmagnetic CVs: the bright spot and the inner disc. It was an historical accident that the first system to be studied in detail was $U$ Gem, in which most of the optical continuum comes from the bright spot, which was shown to be the source of flickering. It would be interesting to see if the amplitude of flickering of the bright spot in CVs in general is roughly a constant fraction of the bright spot luminosity. It would also be useful to try to understand why disc flickering is different from object to object: is this a signature of interaction between inner disc and weakly magnetised primaries?

2. If the hibernation scheme is correct then it is not possible to say that GR provides a lower boundary to the rate of mass transfer - this may be the secular average for short orbital period CVs but the current rate may be as low as zero. The spread of $\dot{M}$ at a given orbital period is probably bounded from the top by saturation of irradiation-driven mass transfer and from the bottom by the selection effect of the necessity of recognising CVs from their disc emissions or outbursts. The full range is easily detected below the $P_{\text {orb }}$ gap - at long periods the secondary dominates and low $\dot{M}$ systems would be difficult currently to find, but they should show up as white dwarf companions to rapidly rotating $\mathrm{K}$ stars.

3. There is increasing evidence, only some of which has been mentioned at this conference, that many supposedly non-magnetic $\mathrm{CV}$ discs are truncated. When discussing the reasons for this it is important to keep all of the evidence in mind, including the behaviour of Dwarf Nova Oscillations during outburst, which I think favours the effects of low magnetic fields in the primaries.

4. The magnetic field geometry in the half dozen polars that have been studied in detail and in the isolated magnetic white dwarfs all depart greatly from simple centred dipoles. They are off-centre dipoles or mixtures of dipole and quadrupole.

The current interpretation, given at this conference, of DQ Her requires a sustained equality of accretion rate onto two magnetic poles. Does this demand a centred dipole geometry? Rosen et al. (1991) point out that in an intermediate polar the effects of asymmetric fields are reduced in the region where gas is threaded by the field lines. Yet at 
this conference we have seen that YY Dra can at times show equally luminous accretion spots and at other times not. Clearly there is more complexity here than had been realised.

5. Deep imaging of old novae has uncovered several shells not before known. Noting the spectacular shell still easily visible in GK Per nearly a century after eruption, it is important to carry out imaging of older novae - i.e. novae that went unnoticed in the nineteenth century which now will be counted among nova-like variables and perhaps even some dwarf novae (noting that some nova remnants have been found to start dwarf nova outbursts within 50 years of nova eruption).

To return to the exponential growth of astronomy, and the popularity of the CV industry. For students hoping to find permanent employment in this research area I point out a book called 'The Perfect CV: How to get the job you really want', by Tom Jackson. The bad news is that it states that 500000 copies have already been sold, so the competition will be fierce.

The Keele Conference was well organised, thanks particularly to the efforts of Janet Wood and Nye Evans, ably supported by C. Hellier, J. Lockley, T. Naylor, F. Ringwald, B. Smalley, M. Somers, W. Welsh and numerous members of the Physics Department.

\section{References}

Rosen, S., Mason, K.O., Mukai, K., Williams, O.R., 1991, MNRAS, 249, 417 